\title{
The neural bases of proactive and reactive control processes in normal aging.
}

\author{
Marine Manard, Caroline Lorant, Eric Salmon, Fabienne Collette \\ Cyclotron Research Centre. University of Liège, Belgium
}

Marine MANARD, Marine.Manard@ulg.ac.be, Allée du VI août, 8 (B30), 4000 Liège, Belgique

Background. Research on cognitive control suggests an age-related decline in proactive control abilities (an anticipatory form of control) whereas reactive control (consecutive to conflict detection) seems to remain intact [1]. As proactive and reactive control abilities were associated to specific brain networks [2], this study investigated age-related effects on the neural substrates associated to each kind of control.

Method. A modified form of the Stroop task was administered to 16 young and 16 older adults in an event-related fMRI experiment. In this version of the Stroop task, three different contexts were created: (1) a mostly congruent context (MC, inducing reactive control) with a majority of congruent items, (2) a mostly incongruent context (MI, inducing proactive control) with mainly interfering items, (3) a neutral context (MN) with mainly neutral items. Preprocessing and statistical analyses were performed with SPM8 ( $\mathrm{p}<.001$ uncorrected).

Results. Behavioral results $(\mathrm{p}<0.05)$ indicated faster processing of interferent items in the MI than MC context in young participants only. With regard to neuroimaging data, the comparison of the two groups showed that the processing of interferent items in the MI context is associated to decreased activity in (mainly right-sided) frontal and temporal areas in the older group. On the contrary, in the MC context, increased activity was observed in bilateral frontal areas for older compared to younger participants.

Discussion. Behavioral results confirm that older participants have difficulties with the implementation of proactive control that is associated to decreased brain activity (compared to young participants) in areas underlying Stroop performance. However, the recruitment of supplementary frontal areas we observed in the reactive control condition suggests compensation processes. So, aging seems to differentially affect the neural networks associated to the various kinds of cognitive control.

(1) Manard et al. (2014). BMC Neuroscience, 15:7

(2) Grandjean et al. (2012). Plos One 7(7), e41513

Keywords cognitive control - executive functions - fMRI- aging 